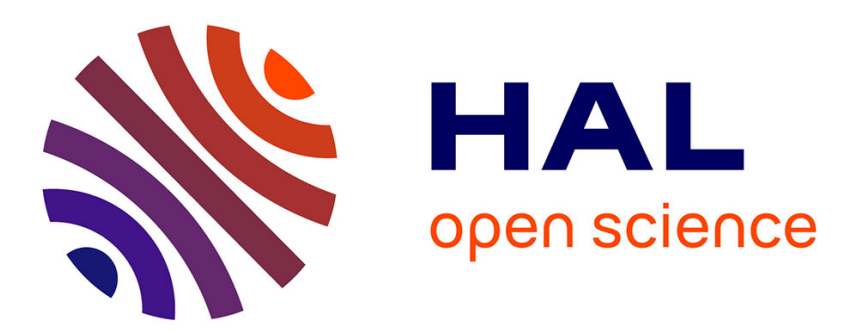

\title{
Quaternion Bessel-Fourier moments and their invariant descriptors for object reconstruction and recognition
} Zhuhong Shao, Huazhong Shu, Jiasong Wu, Beijing Chen, Jean-Louis Coatrieux

\section{- To cite this version: \\ Zhuhong Shao, Huazhong Shu, Jiasong Wu, Beijing Chen, Jean-Louis Coatrieux. Quaternion Bessel- Fourier moments and their invariant descriptors for object reconstruction and recognition. Pattern Recognition, 2014, 47 (2), pp.603-611. 10.1016/j.patcog.2013.08.016 . inserm-00855404}

\section{HAL Id: inserm-00855404 https://www.hal.inserm.fr/inserm-00855404}

Submitted on 29 Aug 2013

HAL is a multi-disciplinary open access archive for the deposit and dissemination of scientific research documents, whether they are published or not. The documents may come from teaching and research institutions in France or abroad, or from public or private research centers.
L'archive ouverte pluridisciplinaire $\mathbf{H A L}$, est destinée au dépôt et à la diffusion de documents scientifiques de niveau recherche, publiés ou non, émanant des établissements d'enseignement et de recherche français ou étrangers, des laboratoires publics ou privés. 


\title{
Quaternion Bessel-Fourier moments and their invariant descriptors for object reconstruction and recognition
}

\author{
Zhuhong Shao ${ }^{1}$, Huazhong Shu ${ }^{1,4}$, Jiasong $\mathrm{Wu}^{1,4}$, Beijing Chen ${ }^{5}$, Jean Louis Coatrieux ${ }^{2,3,4}$ \\ ${ }^{1}$ Laboratory of Image Science and Technology, School of Computer Science and \\ Engineering, Southeast University, 210096 Nanjing, China \\ ${ }^{2}$ INSERM, U1099, Rennes, F-35000, France \\ ${ }^{3}$ Université de Rennes 1, LTSI, Rennes, F-35042, France \\ ${ }^{4}$ Centre de Recherche en Information Médicale Sino-français (CRIBs) \\ ${ }^{5}$ School of Computer \& Software, Nanjing University of Information Science \& Technology, \\ 210044 Nanjing, China
}

\begin{abstract}
In this paper, the quaternion Bessel-Fourier moments are introduced. The significance of phase information in quaternion Bessel-Fourier moments is investigated and an accurate estimation method for rotation angle is described. Furthermore, a new set of invariant descriptors based on the magnitude and the phase information of quaternion Bessel-Fourier moments is derived. Experimental results show that quaternion Bessel-Fourier moments lead to better performance for color image reconstruction than the other quaternion orthogonal moments such as quaternion Zernike moments, quaternion pseudo-Zernike moments and quaternion orthogonal Fourier-Mellin moments. In addition, the angles estimated by the proposed moments are more accurate than those obtained by using other quaternion orthogonal moments. The proposed invariant descriptors show also better robustness to geometric and photometric transformations.
\end{abstract}

Keywords: Quaternion Bessel-Fourier moment, phase, color image, invariant descriptor, recognition 


\section{Introduction}

Moments and moment invariants have been widely used in image processing for pattern recognition [1-3] and image analysis [4-7]. Recently, a new orthogonal moment, the Bessel-Fourier moment, was reported by Xiao [8], wherein the rotation invariance is discussed. However, most of the works reported so far focused on the magnitude coefficients of moments to achieve the rotation invariance and disregarded their corresponding phase information. Unlike the previous works, Li [9] proposed the invariant Zernike moment (ZM) descriptors by combining the ZM magnitude and their phase coefficients as the shape features, which perform better than those described by magnitude-only when applied to noisy image retrieval. Along the same line, another Zernike descriptor preserving the invariance to rotation was described in [10] and used for 2D/3D object recognition. Chen [11] also introduced a ZM phase descriptor, where the ZM magnitude coefficients served as the weighting factors. These works mainly concentrated on gray images or single-channel images.

The quaternion, which can be viewed as the generation of traditional complex number, was introduced by Hamilton in 1843 [12]. The advantage of using the quaternion theory to handle color image is that the existing correlation between color components can be taken into consideration. In the past decades, some quaternion-based techniques have been successfully used for color image processing, for instance the quaternion Fourier transforms $[13,14]$ applied to motion estimation [15] and color image registration [16], the dual-tree quaternion wavelets for multiscale image processing [17]. A set of invariants with respect to geometric transformations (rotation, scaling and translation), based on the magnitude of quaternion Fourier-Mellin moments, has been originally derived by Guo [18]. More recently, the quaternion Zernike moments as well as the quaternion pseudo-Zernike moments and their corresponding invariants were introduced in $[19,20]$, respectively.

Quaternion Bessel-Fourier moments (QBFMs) and their invariant descriptors are here addressed. This paper is organized as follows. Section 2 first provides some preliminaries about 
the conventional Bessel-Fourier moments for gray image and the quaternion theory. The quaternion Bessel-Fourier moments for color image and their algorithms are detailed in section 3. The significance of phase information in the QBFMs is investigated in section 4. Moreover, a new set of quaternion Bessel-Fourier moment descriptors based on angle estimation is specified. Several experiments are carried out in section 5 to show the performance of quaternion Bessel-Fourier moments and the proposed descriptors by contrast with a family of quaternion orthogonal moments including quaternion Zernike moments, quaternion pseudo-Zernike moments and quaternion orthogonal Fourier-Mellin moments as well as their corresponding invariants.

\section{Preliminaries}

In this section, we briefly review the conventional orthogonal Bessel-Fourier moments for gray image and some basic properties of the quaternion.

\subsection{Bessel-Fourier moments}

Considering a gray image $f(\rho, \theta)$ defined in polar coordinates, the Bessel-Fourier moment of order $n$ with repetition $m$ is defined as [8]

$$
B_{n, m}(f)=\frac{1}{2 \pi a_{n}} \int_{0}^{2 \pi} \int_{0}^{1} J_{v}\left(\lambda_{n} \rho\right) f(\rho, \theta) e^{-j m \theta} \rho d \rho d \theta, \quad n=0,1,2, \ldots, m=0, \pm 1, \pm 2, \ldots
$$

where the Bessel functions of the first kind are defined as [21]

$$
J_{v}(x)=\sum_{k=0}^{\infty} \frac{(-1)^{k}}{k ! \Gamma(v+k+1)}\left(\frac{x}{2}\right)^{v+2 k} .
$$

Here $v(v \geq 0)$ is the order of the function and $\Gamma(x)$ the gamma function, $\lambda_{n}$ is the $n$-th zero of the Bessel polynomial and $a_{n}=\left[J_{v+1}\left(\lambda_{n}\right)\right]^{2} / 2$ is the normalization constant.

By using Euler's formula, Eq. (1) can be expressed as

$$
B_{n, m}(f)=\operatorname{Re}\left(B_{n, m}(f)\right)+j \operatorname{Im}\left(B_{n, m}(f)\right),
$$

where $\operatorname{Re}(x)$ represents the real part of the complex $x$, and $\operatorname{Im}(x)$ the imaginary part. 


\subsection{Quaternion}

A quaternion $q$ with one real part and three imaginary parts is given by

$$
q=a+i b+j c+k d
$$

where $a, b, c, d$ are real numbers and $i, j, k$ are orthogonal imaginary units obeying the following rules

$$
i^{2}=j^{2}=k^{2}=-1, i j=-j i=k, j k=-k j=i, k i=-i k=j .
$$

As shown in (5), the quaternion multiplication is not commutative. If $a=0$, then $q=i b+j c+k d$ is called a pure quaternion.

The conjugate of a quaternion $q$ is $\bar{q}=a-i b-j c-k d$. For any two quaternion numbers $p$ and $q$, we have $\overline{p \cdot q}=\bar{q} \cdot \bar{p}$. The quaternion $q$ can also be expressed into polar form as [22]: $q=|q| e^{I \theta}$, where $|q|=\sqrt{a^{2}+b^{2}+c^{2}+d^{2}}$ is called norm, $I=\frac{i b+j c+k d}{\sqrt{b^{2}+c^{2}+d^{2}}}$ and $\theta=\tan ^{-1}\left(\frac{\sqrt{b^{2}+c^{2}+d^{2}}}{a}\right)$ represent the eigenaxis and the eigenangle, respectively.

\section{Quaternion Bessel-Fourier Moments}

\subsection{Definitions}

Let $f(\rho, \theta)$ be an RGB color image defined in polar coordinates. By taking the red, green and blue channels as three imaginary parts, then a quaternion-based model for color image can be represented as

$$
f(\rho, \theta)=i f_{R}(\rho, \theta)+j f_{G}(\rho, \theta)+k f_{B}(\rho, \theta) .
$$

Due to the non-commutative property of quaternion multiplication, there are two types of quaternion Bessel-Fourier moments (QBFMs) based on the Bessel function of the first kind. The right-side QBFMs are defined by

$$
B_{n, m}^{r}(f)=\frac{1}{2 \pi a_{n}} \int_{0}^{2 \pi} \int_{0}^{1} J_{v}\left(\lambda_{n} \rho\right) f(\rho, \theta) e^{-\mu m \theta} \rho d \rho d \theta, \quad n=0,1,2, \ldots, m=0, \pm 1, \pm 2, \ldots
$$


Correspondingly, the left-side QBFMs are given by

$$
B_{n, m}^{l}(f)=\frac{1}{2 \pi a_{n}} \int_{0}^{2 \pi} \int_{0}^{1} e^{-\mu m \theta} J_{v}\left(\lambda_{n} \rho\right) f(\rho, \theta) \rho d \rho d \theta
$$

where $\mu$ is a unit pure quaternion. In this paper, $v$ is set to 1 and $\mu=(i+j+k) / \sqrt{3}$.

According to the anti-involution property of quaternion conjugation, the left-side and right-side QBFMs for the same color image have the following relationship

$$
\begin{aligned}
& B_{n, m}^{l}(f)=\overline{\frac{1}{2 \pi a_{n}} \int_{0}^{2 \pi} \int_{0}^{1} e^{-\mu m \theta} J_{v}\left(\lambda_{n} \rho\right) f(\rho, \theta) \rho d \rho d \theta} \\
& =-\overline{\frac{1}{2 \pi a_{n}} \int_{0}^{2 \pi} \int_{0}^{1} J_{v}\left(\lambda_{n} \rho\right) f(\rho, \theta) e^{\mu m \theta} \rho d \rho d \theta} \\
& =-\overline{B_{n,-m}^{r}(f)} \text {. }
\end{aligned}
$$

In the following sections, the QBFMs refer to the right-side type.

It can be easily verified that the inverse transform of (7) is given by

$$
f(\rho, \theta)=\sum_{n=0}^{\infty} \sum_{m=-n}^{n} J_{v}\left(\lambda_{n} \rho\right) B_{n, m}^{r}(f) e^{\mu m \theta}
$$

When only a finite number of the QBFMs with order up to $P$ is used, (10) is approximated by

$$
f^{q}(\rho, \theta)=\sum_{n=0}^{P} \sum_{m=-n}^{n} J_{v}\left(\lambda_{n} \rho\right) B_{n, m}^{r}(f) e^{\mu m \theta}
$$

For digital images, the double integral in Eq. (7) is substituted by a double summation. Its discrete form is given by

$$
B_{n, m}^{r}(f)=\frac{1}{2 \pi a_{n}(N-1)^{2}} \sum_{x=1}^{N} \sum_{y=1}^{N} J_{v}\left(\lambda_{n} \rho\right) f(x, y) e^{-\mu m \theta}
$$

where $N$ is the number of pixels in each coordinate axis of the image, the parameters $\rho$ and $\theta$ are computed by the mapping transformation as follows [7]

$$
\rho=\sqrt{\left(c_{1} x+c_{2}\right)^{2}+\left(c_{1} y+c_{2}\right)^{2}}, \theta=\tan ^{-1}\left(\frac{c_{1} y+c_{2}}{c_{1} x+c_{2}}\right), c_{1}=\frac{\sqrt{2}}{N-1}, c_{2}=-\frac{1}{\sqrt{2}} .
$$

\subsection{Algorithm}


Substituting (6) into (7) and combining (3), we have

$$
\begin{aligned}
& B_{n, m}^{r}(f) \\
= & \frac{1}{2 \pi a_{n}} \int_{0}^{2 \pi} \int_{0}^{1} J_{v}\left(\lambda_{n} \rho\right) f(\rho, \theta) \mathrm{e}^{-\mu m \theta} \rho d \rho d \theta \\
= & \frac{1}{2 \pi a_{n}} \int_{0}^{2 \pi} \int_{0}^{1} J_{v}\left(\lambda_{n} \rho\right)\left(i f_{R}(\rho, \theta)+j f_{G}(\rho, \theta)+k f_{B}(\rho, \theta)\right)(\cos (m \theta)-\mu \sin (m \theta)) \rho d \rho d \theta \\
= & i\left[\operatorname{Re}\left(B_{n, m}\left(f_{R}\right)\right)+\mu \operatorname{Im}\left(B_{n, m}\left(f_{R}\right)\right)\right]+j\left[\operatorname{Re}\left(B_{n, m}\left(f_{G}\right)\right)+\mu \operatorname{Im}\left(B_{n, m}\left(f_{G}\right)\right)\right]+k\left[\operatorname{Re}\left(B_{n, m}\left(f_{B}\right)\right)+\mu \operatorname{Im}\left(B_{n, m}\left(f_{B}\right)\right)\right] \\
= & A_{n, m}+i X_{n, m}+j Y_{n, m}+k Z_{n, m},
\end{aligned}
$$

where

$$
\begin{aligned}
& A_{n, m}=\frac{-1}{\sqrt{3}}\left[\operatorname{Im}\left(B_{n, m}\left(f_{R}\right)\right)+\operatorname{Im}\left(B_{n, m}\left(f_{G}\right)\right)+\operatorname{Im}\left(B_{n, m}\left(f_{B}\right)\right)\right], \\
& X_{n, m}=\operatorname{Re}\left(B_{n, m}\left(f_{R}\right)\right)+\frac{1}{\sqrt{3}}\left[\operatorname{Im}\left(B_{n, m}\left(f_{G}\right)\right)-\operatorname{Im}\left(B_{n, m}\left(f_{B}\right)\right)\right], \\
& Y_{n, m}=\operatorname{Re}\left(B_{n, m}\left(f_{G}\right)\right)+\frac{1}{\sqrt{3}}\left[\operatorname{Im}\left(B_{n, m}\left(f_{B}\right)\right)-\operatorname{Im}\left(B_{n, m}\left(f_{R}\right)\right)\right], \\
& Z_{n, m}=\operatorname{Re}\left(B_{n, m}\left(f_{B}\right)\right)+\frac{1}{\sqrt{3}}\left[\operatorname{Im}\left(B_{n, m}\left(f_{R}\right)\right)-\operatorname{Im}\left(B_{n, m}\left(f_{G}\right)\right)\right] .
\end{aligned}
$$

Here $B_{n, m}\left(f_{R}\right), B_{n, m}\left(f_{G}\right)$ and $B_{n, m}\left(f_{B}\right)$ are the conventional Bessel-Fourier moments of the red, green and blue channels, respectively. From (14), one can conclude that the computation of QBFMs can be implemented via the conventional Bessel-Fourier moments of three single-channel images.

To compute one $B_{n, m}^{r}(f)$ of an RGB image $f(\rho, \theta)$ with $N \times N$ pixels, the proposed algorithm using (14) only needs $3 N^{2}+5$ additions and $6 N^{2}+4$ multiplications while the direct algorithm based on (7) requires $9 N^{2}-1$ additions and $12 N^{2}+1$ multiplications.

\section{The invariant quaternion Bessel-Fourier moment descriptors}

\subsection{The significance of phase in quaternion moments}

As pointed out in [23], the signal phase in Fourier domain plays an important role. Here, its significance in the QBFMs for color image is investigated. The color image Lion king cup, with size $144 \times 192$ (shown in Fig. 1 (a)) selected from Amsterdam library of object images [24], was used for reconstruction purpose using QBFMs of order up to 9 with and without the phase 
information. It can be seen from Fig. 1 that the phase coefficients of the QBFMs not only embody the contour but also the color components. Theoretically, when only magnitude coefficients of the QBFMs are used for reconstructing color image, according to the odd-even property, (11) can be simplified as

$$
f^{\prime}(\rho, \theta)=2 \sum_{n=0}^{P} \sum_{m=0}^{n} J_{v}\left(\lambda_{n} \rho\right)\left|B_{n, m}^{r}(f)\right| \cos (m \theta) .
$$

The result of (15) is a real matrix, in other words, it is a gray image as displayed in Fig. 1, where the color information cannot be retrieved. The same conclusions can also be drawn for other quaternion orthogonal moments such as quaternion Zernike moments, quaternion pseudo-Zernike moments and quaternion orthogonal Fourier-Mellin moments.

\subsection{The proposed invariant descriptors}

From the above analysis, the phase information in quaternion Bessel-Fourier moments appears as more informative when compared to their corresponding magnitude coefficients. In [11], the ZM phase descriptor for gray image or single-channel image was introduced. To deal with a rotated color image in a whole vector, a robust estimation method of rotated angles through quaternion Bessel-Fourier moments is presented and the invariant descriptors are also introduced.

Let $f_{r}(\rho, \theta)$ represent the reference image and $f_{t}(\rho, \theta)$ the transformed image with rotation angle $\alpha$, respectively. The relationship between the QBFMs of the two images is given by

$$
B_{n, m}^{r}\left(f_{t}\right)=B_{n, m}^{r}\left(f_{r}\right) e^{-\mu m \alpha},
$$

where $m \geq 1$ and $\alpha \in[0,2 \pi]$. Like the traditional Bessel-Fourier moments, the QBFM magnitudes are invariant to image rotation, i.e., $\left|B_{n, m}^{r}\left(f_{t}\right)\right|=\left|B_{n, m}^{r}\left(f_{r}\right)\right|$. Meanwhile, the phase difference is

$$
\phi_{n, m}=(m \alpha) \bmod (2 \pi), \phi_{n, m} \in(0,2 \pi]
$$

where 


$$
m \alpha=\arg \left(\frac{1}{B_{n, m}^{r}\left(f_{t}\right)} B_{n, m}^{r}\left(f_{r}\right)\right) \neq \arg \left(\frac{B_{n, m}^{r}\left(f_{r}\right)}{B_{n, m}^{r}\left(f_{t}\right)}\right) .
$$

The symbol ' $f$ ' is due to the noncommutativity of quaternion multiplication, and $\arg (\cdot)$ denotes the phase of quaternion.

By using any two adjacent $\phi_{n, m-1}$, and $\phi_{n, m}(m \neq 0)$, the rotation angle $\alpha$ can be estimated as follows

$$
\begin{aligned}
\alpha & =\left(\phi_{n, m}+2 k_{n, m} \pi\right)-\left(\phi_{n, m-1}+2 k_{n, m-1} \pi\right) \\
& =\left(\phi_{n, m}-\phi_{n, m-1}\right) \bmod (2 \pi) .
\end{aligned}
$$

As pointed out by Chen and Sun [11], there are many ways to calculate the rotation angle. They proposed a robust approach to obtain the angle value which consists of weighting the estimated angles by the individual magnitude of the moments. Here, we adopt the same strategy: an iterative algorithm to compute the rotation angle $\hat{\alpha}$ weighted by the individual magnitude $\left|B_{n, m}^{r}\right|$ with different orders $(n, m)$ is described in Fig. 2. For more details, we refer to [11].

For the reference image $f_{r}(\rho, \theta)$ and its transformed image $f_{t}(\rho, \theta)$, if there exists a rotation angle $\alpha$ between them, then $\left|\phi_{n, m}-(m \hat{\alpha}) \bmod (2 \pi)\right|$ is equal to 0 , otherwise $\left|\phi_{n, m}-(m \hat{\alpha}) \bmod (2 \pi)\right|$ has a nonzero value ranging from 0 to $2 \pi$, where $\hat{\alpha}$ represents a putative angle and is estimated through the proposed algorithm. Then, the normalized similarity distance based on phase information of quaternion Bessel-Fourier moments is defined as

$$
d^{\text {ang }}\left(f_{r}, f_{t}\right)=\sum_{m} \sum_{n} \frac{\min \left\{\left|\phi_{n, m}-(m \hat{\alpha}) \bmod (2 \pi)\right|, 2 \pi-\left|\phi_{n, m}-(m \hat{\alpha}) \bmod (2 \pi)\right|\right\}}{\pi} .
$$

Combining the normalized magnitude coefficients that will serve as weighting factors, a similarity measure based on the weighted and normalized phase descriptors can be defined as

$$
d\left(f_{r}, f_{t}\right)=\sum_{m} \sum_{n} \omega_{n, m}^{\prime} \frac{\min \left\{\left|\phi_{n, m}-(m \hat{\alpha}) \bmod (2 \pi)\right|, 2 \pi-\mid \phi_{n, m}-(m \hat{\alpha}) \bmod (2 \pi)\right\}}{\pi}
$$

where 


$$
\omega_{n, m}^{\prime}=\frac{\left|B_{n, m}^{r}\left(f_{r}\right)\right|+\left|B_{n, m}^{r}\left(f_{t}\right)\right|}{\sum_{n, m}\left(\left|B_{n, m}^{r}\left(f_{r}\right)\right|+\left|B_{n, m}^{r}\left(f_{t}\right)\right|\right)} .
$$

The similarity measure $d\left(f_{r}, f_{t}\right)$ defined in (20) is quasi invariant to image rotation. In fact, when an image is rotated with an angle $\alpha$, the weighting factors $\omega_{n, m}^{\prime}$ are independent to image rotation because only the magnitude is used in their definition. On the other hand, the estimated value $m \hat{\alpha} \bmod 2 \pi$ obtained by the proposed approach is very close to the true value $\phi_{n, m}$. That means that the value of the numerator in (20) is nearly equal to zero.

When an image undergoes both rotation and translation, the translation invariance can be achieved by locating the origin of the coordinate system at the common centroid defined in [25]. Thus the proposed descriptors are invariant to both image rotation and translation. However, they are not invariant to image scaling. To obtain the scaling invariance, the normalization process is required. Notice that the algorithm described above can also be applied to other quaternion moments such as quaternion Zernike moments, quaternion pseudo-Zernike moments or quaternion orthogonal Fourier-Mellin moments.

\section{Experimental results}

To illustrate the interest of the quaternion Bessel-Fourier moments for color image reconstruction and the robustness of proposed descriptors against geometric and photometric transformations, some experiments were carried out. As aforementioned, both the quaternion Zernike moments and the quaternion pseudo-Zernike moments and their corresponding invariants have been reported in [19] and [20], respectively. To make a full comparison in the following experiments, we also extended the conventional orthogonal Fourier-Mellin moments originally reported in [3] for gray image to the quaternion domain for color image (named quaternion orthogonal Fourier-Mellin moments hereafter) and derived their corresponding invariants by using the approach developed for quaternion Zernike moment-based and quaternion 
pseudo-Zernike moment-based invariants (for more details, we refer to [19] and [26]).

\subsection{Color image reconstruction}

This experiment was conducted to evaluate the reconstruction capability of QBFMs for color images. The tested images consist of 100 color images with size $144 \times 192$ (images samples are shown in Fig. 3) selected from Amsterdam library of object images [24]. The standard normalized image reconstruction error (NIRE) is used to compute the difference between the original image $f(x, y)$ and the reconstructed image $f(0 x, y) \quad[3]$

$$
N I R E=\frac{\sum_{x=0}^{N-1} \sum_{y=0}^{N-1}|f(x, y)-f(p x, y)|^{2}}{\sum_{x=0}^{N-1} \sum_{y=0}^{N-1}|f(x, y)|^{2}} .
$$

The comparison of NIRE values obtained with different orders of quaternion Zernike moments (QZMs) [19], quaternion pseudo-Zernike moments (QPZMs) [20] and quaternion orthogonal Fourier-Mellin moments (QOFMMs) is shown in Fig. 4. For each of 100 tested color images, 19 reconstructed images are obtained by using any one of four quaternion moments, where the moment orders are from 3 to 57 with an increment 3 , resulting in total $7600(=100 \times 19 \times 4)$ reconstructed images and some of them are shown in Fig. 5. It can be clearly seen that: (1) the value of NIRE decreases when increasing the moment order, and the error values corresponding to the QBFMs are always smallest; (2) the inspection of the reconstructed images shows that the QBFMs with $P=48$ lead to a better visual restitution of the object (this qualitative analysis is fully coherent with the NIRE quantitative index where the performance of QOFMMs is the closest to QBFMs).

\subsection{Accuracy of rotation angle estimation}

The accuracy of rotation angle estimation by the QBFMs was tested in another experiment and 
compared with the ones obtained with the QZMs, the QPZMs and the QOFMMs. The set of 100 color images employed in section 5.1 were reused. To obtain the entire transformed image after rotation, the actual size of tested images in this section is $256 \times 256$ by zero padding. All the images were rotated from $10^{\circ}$ to $350^{\circ}$ with an increment $10^{\circ}$, forming a set of 35 tested images for each sample. The rotation angles were estimated on the $3500(=100 \times 35)$ resulting images using the proposed QBFMs, the QZMs, the QPZMs and the QOFMMs, respectively. The root mean square (RMS) error $e_{1}$ and the maximum error $e_{2}$ of estimated angles are defined as [27]

$$
\begin{gathered}
e_{1}=\sqrt{\frac{1}{M} \sum_{k=1}^{M}\left(\theta_{k}-\theta_{k}\right)^{2}} \\
e_{2}=\max \left\{\mid \theta_{k}-\theta_{k} \phi, 1 \leq k \leq M\right\},
\end{gathered}
$$

where $M$ is the number of rotation angles, $\theta_{k}$ is the rotation angle and $\theta_{k}^{0}$ the estimated one.

The average RMS error and maximum error are listed in Table 1, where the numbers of QBFMs are respectively 21 (with order from 1 to 6), 28 (with order from 1 to 7), 36 (with order from 1 to 8), while the numbers of QZMs are 20 (with order from 1 to 8), 30 (with order from 1 to 10 ), 36 (with order from 1 to 11 ) and the numbers of QPZMs and QOFMMs are the same as those of QBFMs. Note that the moments with repetition $\mathrm{m}=0$ have not been used because they are not affected by image rotation. Note also that the constraint $|\mathrm{m}| \leq \mathrm{n}$ was imposed for both QBFMs and QOFMMs in order to ensure that the same number of moments can be chosen as that of QPZMs. It can be seen that: (1) the estimated angles become more accurate as the number of QBFMs increases, the same phenomenon is also observed for the QZMs, the QPZMs and the QOFMMs; (2) both the RMS error and maximum error of estimated angles by using the QBFMs are much smaller. These results indicate that the rotation angles estimated by the proposed QBFMs are more accurate than those obtained with other quaternion orthogonal moments.

\subsection{Object recognition}

To test the robustness of the proposed QBFM descriptors (QBFMD) against geometric 
(rotation, scaling and translation, RST) and photometric (noise and blur) transformations, a set of 100 color images selected from Columbia University Image Library [28] with size $86 \times 86$ was used in this experiment (image samples are shown in Fig. 6). To obtain the entire transformed image after rotation, the tested images have been zero padded to get a $128 \times 128$ format. A comparison with the quaternion moment-based invariants including quaternion Zernike moment invariants (QZMIs), quaternion pseudo-Zernike moment invariants (QPZMIs) and quaternion orthogonal Fourier-Mellin moment invariants (QOFMMIs) was conducted.

The testing set is then constructed as follows: each sample image was scaled with the factor $\lambda$ $\in\{0.5,1.0,1.5\}$, and rotated from $15^{\circ}$ to $255^{\circ}$, with an increment $30^{\circ}$; then each image was translated by $(\Delta x, \Delta y)=(3,5)$ (other values can also be chosen). The final RST testing set is therefore composed of $2700(=100 \times 3 \times 9 \times 1)$ images. To test the robustness of different descriptors, we have also added Gaussian noise (zero mean and varying standard deviation $\sigma$ ), smooth distortions with variable size $s$, and salt-and-pepper noise with different densities $d$ to the testing set. Some examples of tested images corrupted by different types of noise or smooth distortions are displayed in Fig. 7. The nearest neighbor classifier based on Euclidean distance is used to measure the correct classification percentage (CCP), which is defined by

$$
\eta=\frac{\text { Number of correctly classified images }}{\text { Total number of used images in testing set }} \times 100 \% \text {. }
$$

Note that, because the proposed descriptors are only invariant to image translation and rotation, the normalization process is required when image scaling is concerned, such a process, however, is not necessary for other invariant descriptors. The recognition results are depicted in Fig. 8, where the central QBFMs are used for the proposed QBFMD and the number of the moments is respectively 21 and 36. For comparison purpose, we have chosen 21 central QZMIs defined in [19] among 25 of order up to 8, and 36 of order up to 10; 21 central QPZMIs defined in [20] of order up to 5, and 36 of order up to 7; and 21 central QOFMMIs among 25 of order up to 5, and 36 of order up to 7, respectively. From these results, it can be seen that: (1) When the number is 
21, the QBFMDs provide higher recognition accuracy under RST transformations, additive noise with different levels and smooth distortions with variable size; (2) When increasing the number up to 36, an important feature also observed is that the performance of QBFMDs remains stable with the increase of the noise level while the behavior of other three quaternion moment-based invariants degrades with different degrees. The results show clearly that the proposed descriptors could be useful as a new feature descriptor for object recognition.

\section{Conclusion}

This paper has introduced the quaternion Bessel-Fourier moments in order to improve image reconstruction and pattern recognition by simultaneously handling the three image color channels. The significance of phase information in quaternion Bessel-Fourier moments has been investigated and an accurate estimation method for rotation angle throughout the new moments has been proposed. Furthermore, a new set of invariant descriptors, not only using the magnitude coefficients of quaternion Bessel-Fourier moments but also their corresponding phase information, have been elaborated and applied to color object recognition for validation. Experimental results show that the quaternion Bessel-Fourier moments have a better performance in terms of color image reconstruction than the other quaternion orthogonal moments such as quaternion Zernike moments, quaternion pseudo-Zernike moments and quaternion orthogonal Fourier-Mellin moments. In addition, the estimated angles by quaternion Bessel-Fourier moments are more accurate than those obtained by other three quaternion orthogonal moments employed above and the recognition accuracy indicates that the quaternion Bessel-Fourier moment descriptors are more robust against geometric and photometric transformations when compared to quaternion moment-based invariants such as quaternion Zernike moment invariants, quaternion pseudo-Zernike moment invariants as well as quaternion orthogonal Fourier-Mellin moment invariants. Future work will concentrate on the application of the proposed descriptors to other color image processing area such as texture analysis and registration. 


\section{Acknowledgement}

This work was supported by the National Basic Research Program of China under Grant 2011CB707904, by the National Natural Science Foundation of China under Grants 61073138, $61103141,61271312,61201344$, and 81101104 , by the Ministry of Education of China under Grants 20110092110023 and 20120092120036, the Key Laboratory of Computer Network and Information Integration (Southeast University), Ministry of Education, and by Natural Science Foundation of Jiangsu Province under Grants SBK200910055, BK2012329, and BK2012743. The authors would like to thank the anonymous reviewers for their constructive comments and suggestions, which have greatly helped us in improving the quality of the paper.

\section{References}

[1] M.K. Hu, Visual pattern recognition by moment invariants, IRE Trans. Inf. Theory 8 (2) (1962) 179-187.

[2] A. Khotanzad, Y.H. Hong, Invariant image recognition by Zernike moments, IEEE Trans. Pattern Anal. Mach. Intell. 12 (5) (1990) 489-497.

[3] Y.L. Sheng, L.X. Shen, Orthogonal Fourier-Mellin moments for invariant pattern recognition, J. Opt. Soc. Am. A 11 (6) (1994) 1748-1757.

[4] C.H. Teh, R.T. Chin, On image analysis by the methods of moments, IEEE Trans. Pattern Anal. Mach. Intell. 10 (4) (1988) 496-513.

[5] H.Z. Shu, L.M. Luo, J.L. Coatrieux, Moment-based approaches in image Part 1: basic features, IEEE Eng. Med. Biol. Mag. 26 (2007) 70-74.

[6] H.Z. Shu, L.M. Luo, J.L. Coatrieux, Moment-based approaches in image Part 2: invariance, IEEE Eng. Med. Biol. Mag. 27 (2008) 81-83.

[7] C.W. Chong, P. Raveendran, R. Mukundan, Translation invariants of Zernike moments, Pattern Recognition. 36 (8) (2003) 1765-1773. 
[8] B. Xiao, J.F. Ma, W. Xuan, Image analysis by Bessel-Fourier moments, Pattern Recognition. 43 (8) (2010) 2620-2629.

[9] S. Li, M. C. Lee, C.M. Pun, Complex Zernike moment features for shape-based image retrieval, IEEE Trans. Syst. Man Cyb. A 39 (1) (2009) 227-237.

[10] J. Revaud, G. Lavoué, A. Baskurt, Improving Zernike moments comparison for optical similarity and rotation angle retrieval, IEEE Trans. Pattern Anal. Mach. Intell. 31 (4) (2009) $627-636$.

[11] Z. Chen, S.K. Sun, A Zernike moment phase-based descriptor for image representation and matching, IEEE. Trans. Image Process. 19 (1) (2010) 205-219.

[12] W.R. Hamilton, Elements of quaternions, Longmans Green, London, U.K., 1866.

[13] T.A. Ell, Hypercomplex Spectral Transforms, Ph.D. dissertation, Univ. Minnesota, Minneapolis, 1992.

[14] T.A. Ell, S.J. Sangwine, Hypercomplex Fourier transforms of color images, IEEE Trans. Image Process. 16 (1) (2007) 22-35.

[15] D.S. Alexiadis, G.D. Sergiadis, Estimation of motions in color image sequences using hypercomplex Fourier transforms, IEEE Trans. Image Process. 18 (1) (2009) 168-187.

[16] C.E. Moxey, S.J. Sangwine, T.A. Ell, Color-grayscale image registration using hypercomplex phase correlation, in: Proceedings of the IEEE International Conference on Image Processing (ICIP), 2002, pp. 385-388.

[17] W.L. Chan, H. Choi, R.G. Baraniuk, Coherent multiscale image processing using dual-tree quaternion wavelets, IEEE Trans. Image Process. 17 (7) (2008) 1069-1082.

[18] L.Q. Guo, M. Zhu, Quaternion Fourier-Mellin moments for color image, Pattern Recognition. 44 (2) (2011) 187-195.

[19] B.J. Chen, H.Z. Shu, H. Zhang, G. Chen, C. Toumoulin, J.L. Dillenseger, L.M. Luo, Quaternion Zernike moments and their invariants for color image analysis and object recognition, Signal Processing. 92 (2) (2012) 308-318. 
[20] B.J. Chen, X.M. Sun, D.C. Wang, X.P. Zhao, Color face recognition using quaternion representation of color image, ACTA Automatica Sinica 38 (11) (2012) 1815-1823.

[21] M. Abramowitz, I.A. Stegun, Handbook of Mathematical Functions, National Bureau of Standards, Applied Mathematics Series, vol. 55, Dover Publications, 1965.

[22] T.A. Ell, Quaternion-Fourier transforms for analysis of two-dimensional linear time-invariant partial differential systems, in: IEEE Proceedings of the 32nd Conference on Decision and Control, 1993, pp. 1830-1841.

[23] A.V. Oppenheim, J.S. Lim, The importance of phase in signals, Proc. IEEE 69 (5) (1981) 529-541.

[24] J. M. Geusebroek, G. J. Burghouts, A. W. M. Smeulders, The Amsterdam library of object images, Int. J. Comput. Vision 61 (1) (2005) 103-112

[25] T. Suk, J. Flusser, Affine moment invariants of color images, in: Proceedings of 13th International Conference on Computer Analysis of Images and Patterns (CAIP), 5702 (2009), pp. 334-341.

[26] H. Zhang, H.Z. Shu, P. Haigron, B.S. Li, L.M. Luo, Construction of a complete set of orthogonal Fourier-Mellin moment invariants for pattern recognition applications, Image Vision Comput. 28 (2010) 38-44.

[27] W.Y. Kim, Y.S. Kim, Robust rotation angle estimator, IEEE Trans. Pattern Anal. Mach. Intell. $21(8)(1999) 768-773$.

[28] < http://www.cs.columbia.edu/CAVE/software/softlib/coil-100.php > 


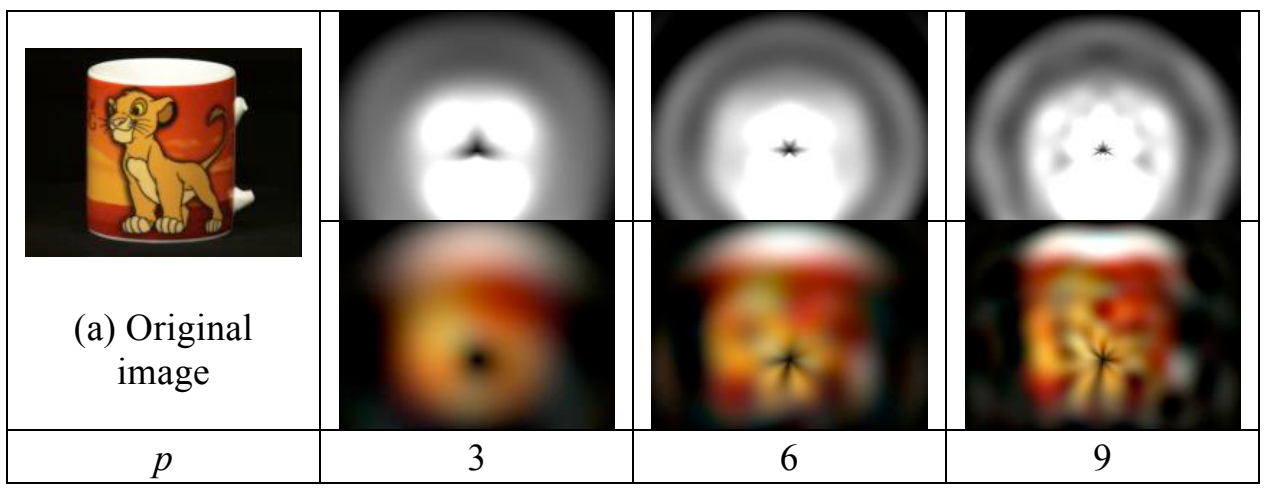

Fig. 1. Examples of the reconstructed images by the QBFMs: the first row uses magnitudes alone, the second one contains phase coefficients. The moment orders range from 3 to 9 .

$$
\begin{aligned}
& \text { start: } \hat{\alpha}_{0}=0 ; c_{0}=0 \\
& \text { for } m=1,2, \ldots, P \\
& \text { for } n=m, m+1 \ldots, P \\
& \delta_{n, m}=\left(\phi_{n, m}-(m-1) \hat{\alpha}_{m-1}\right) \bmod (2 \pi) \\
& \omega_{n, m}=\frac{\left|B_{n, m}^{r}\left(f_{t}\right)\right|+\left|B_{n, m}^{r}\left(f_{r}\right)\right|}{2} \\
& \text { end } \\
& s_{m}=\sum_{n=m}^{P} \frac{\omega_{n, m}}{m} \\
& \delta_{m}=\frac{1}{s_{m}} \sum_{n=m}^{P} \frac{\omega_{n, m}}{m} \delta_{n, m} \\
& \hat{\alpha}_{m}=\frac{1}{c_{m-1}+s_{m}}\left(c_{m-1} \hat{\alpha}_{m-1}+s_{m} \delta_{m}\right) \\
& c_{m}=c_{m-1}+s_{m} \\
& \text { end } \\
& \hat{\alpha}=\hat{\alpha}_{P}
\end{aligned}
$$

Fig. 2. The algorithm for computing the rotation angle 


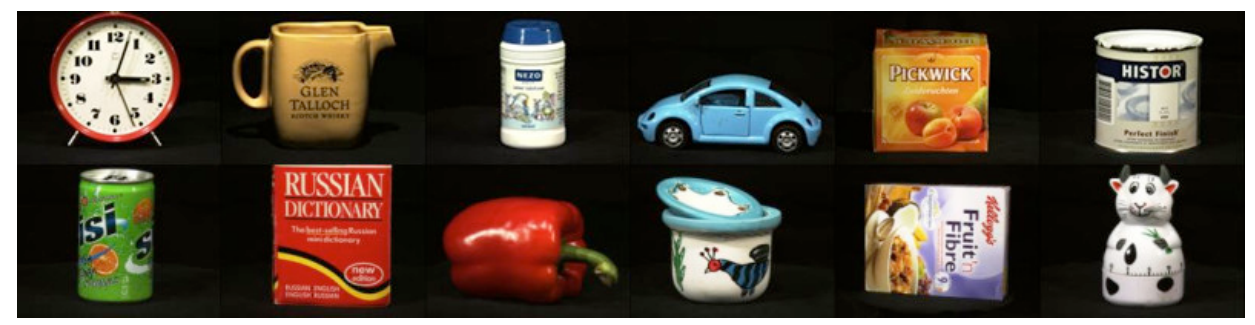

Fig. 3. Some examples of color images selected from Amsterdam Library of Object Images [24] for reconstruction

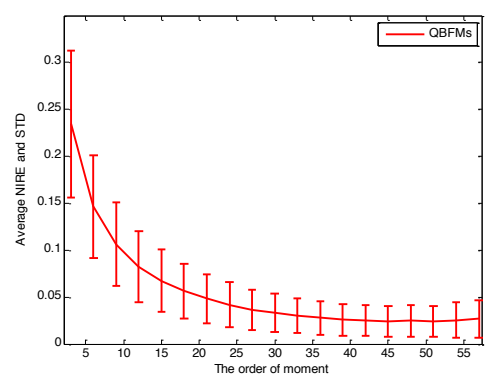

(a)

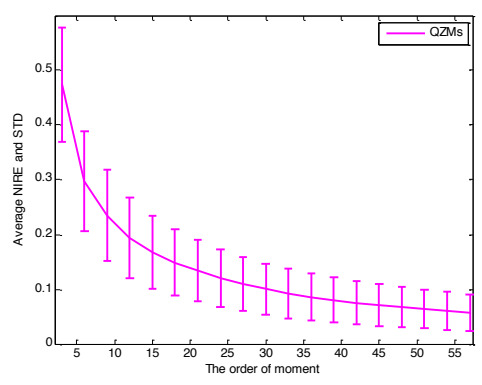

(b)

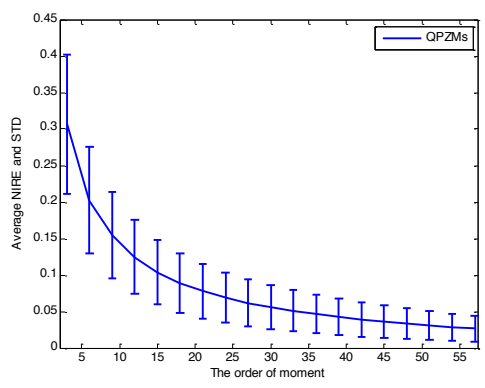

(c)

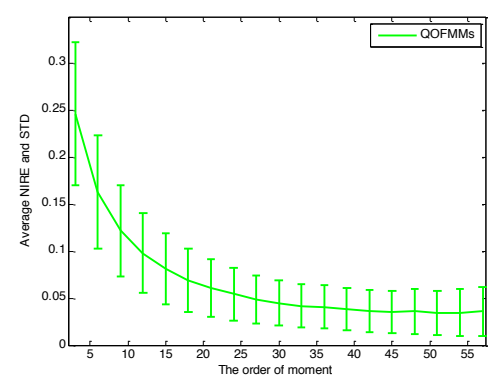

(d)

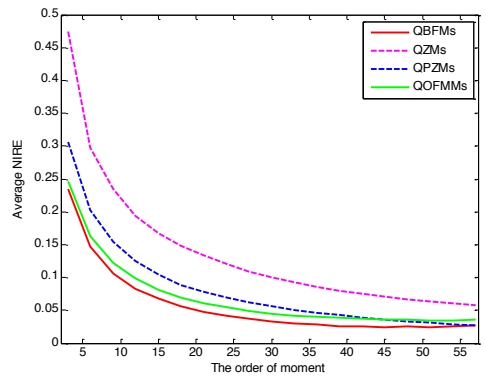

(e)

Fig. 4. NIRE values of reconstructed image for quaternion Bessel-Fourier moments (QBFMs), quaternion Zernike moments (QZMs), quaternion pseudo-Zernike moments (QPZMs) and quaternion orthogonal Fourier-Mellin moments (QOFMMs): (a)-(d) show the average and stand deviations (STD) of NIRE, (e) show the overall average NIRE. 


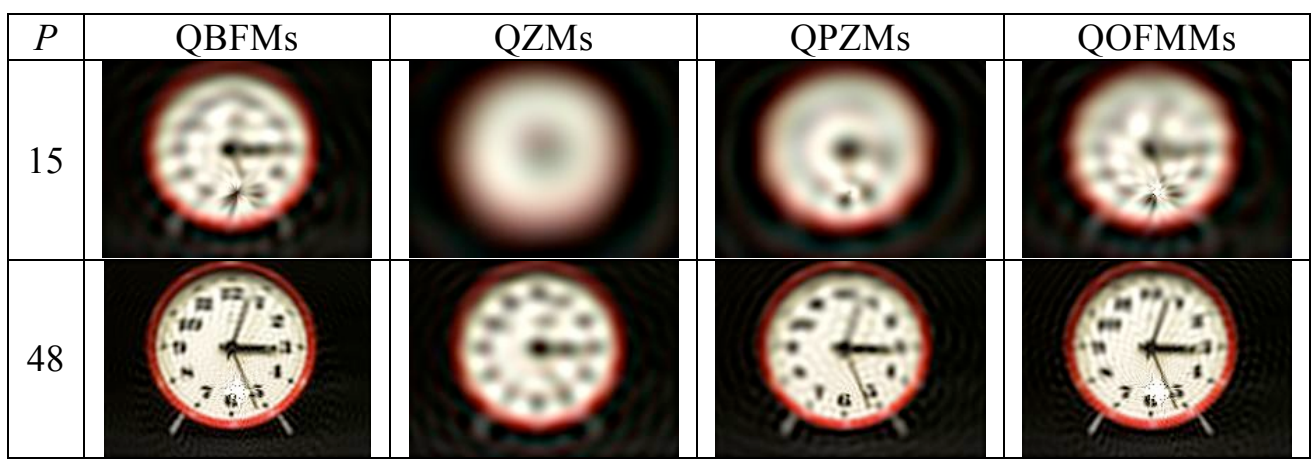

Fig. 5. Examples of reconstructed image by different types of quaternion moments

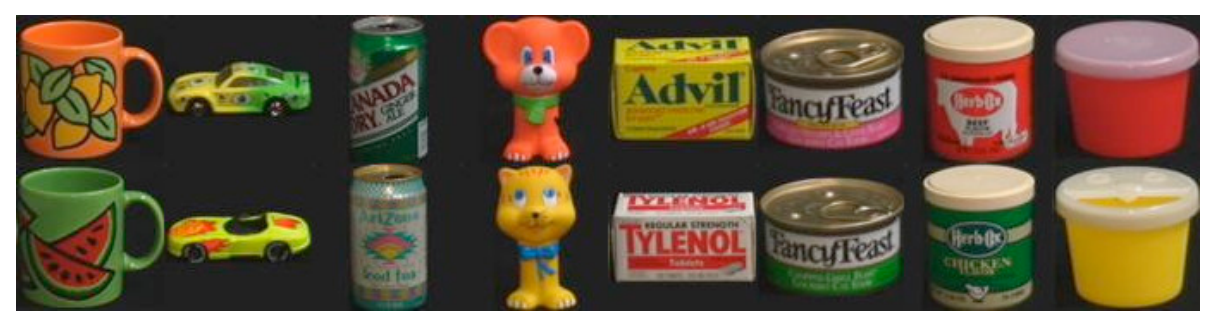

Fig. 6. Some examples of color images selected from Columbia University Image Library [28] 


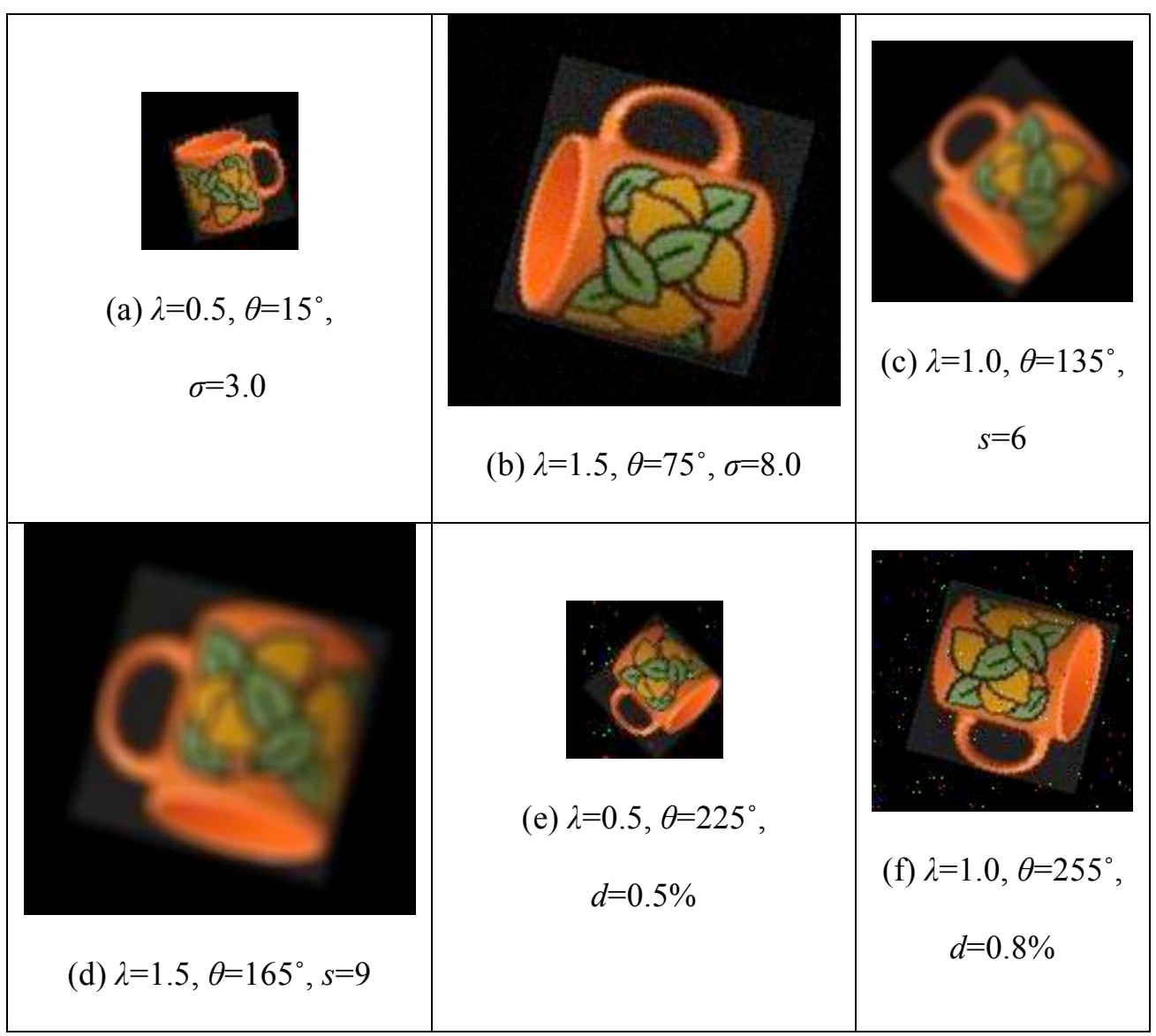

Fig. 7. Examples of image with different noise and smooth distortions: (a) and (b) correspond to Gaussian noise, (c) and (d) to smooth distortion, (e) and (f) to salt-and-pepper noise. 


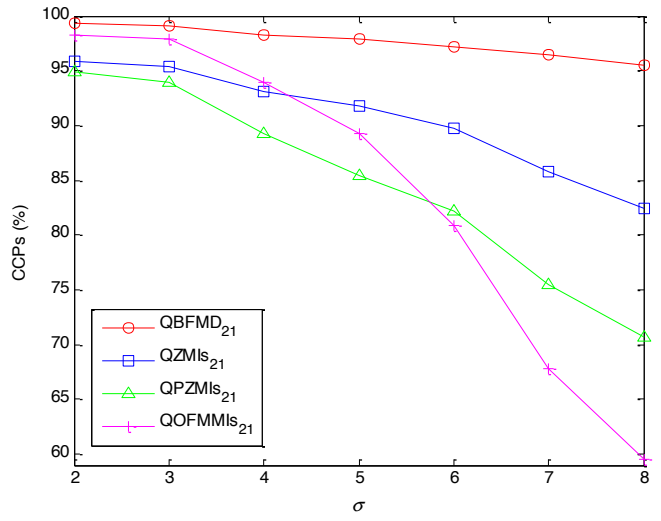

(a)

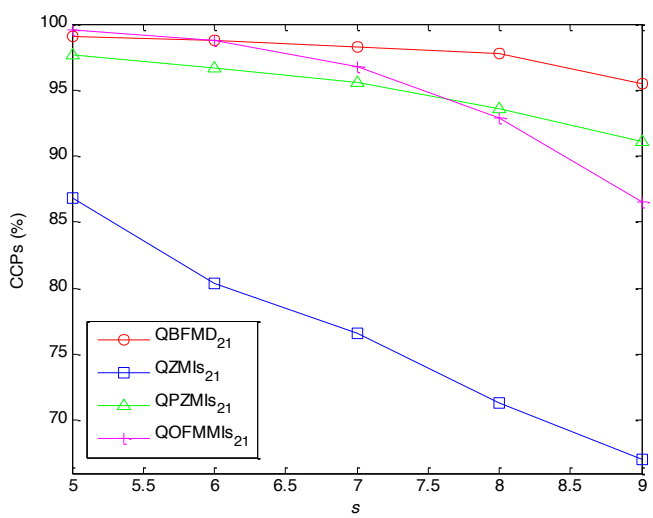

(c)

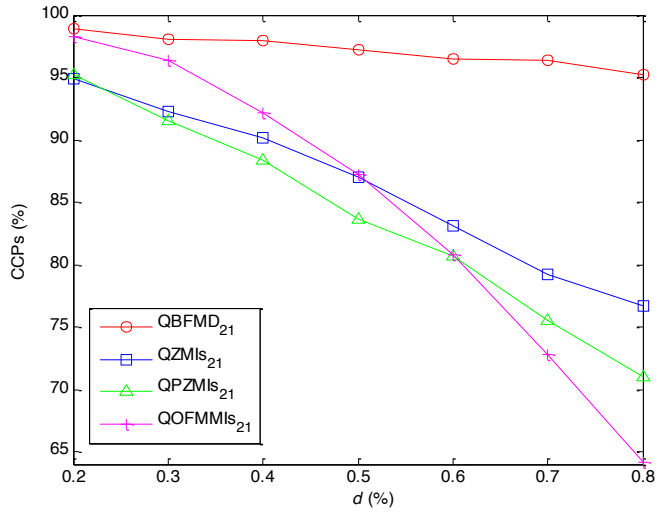

(e)

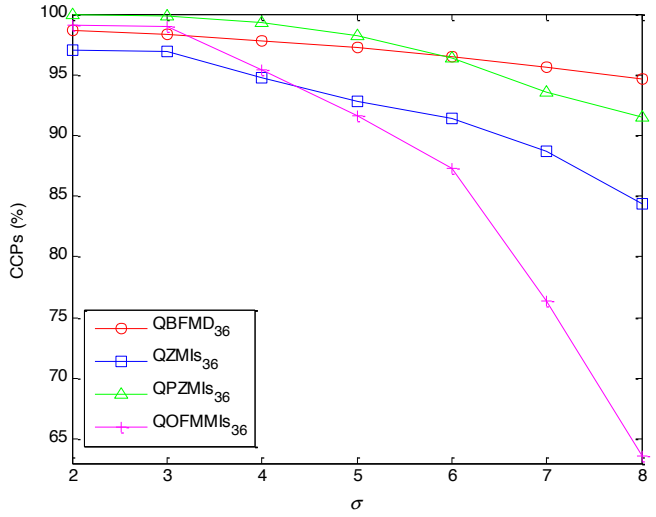

(b)

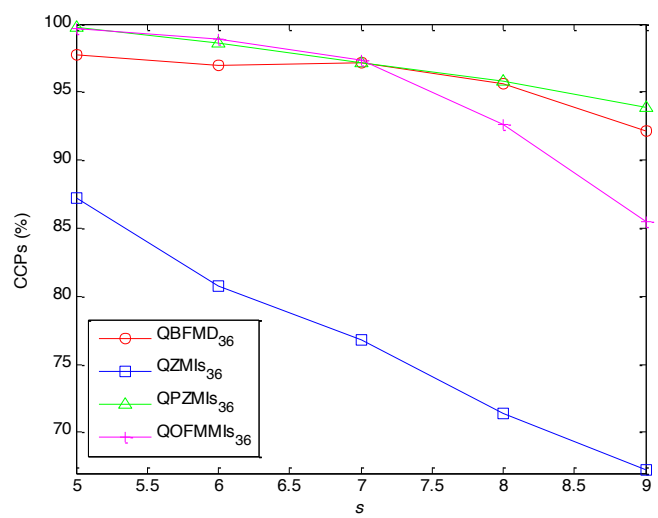

(d)

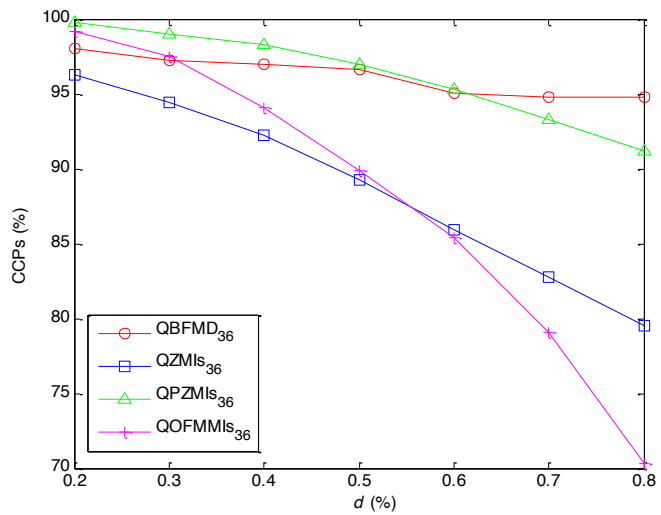

(f)

Fig. 8. Comparison of $\mathrm{CCP}$ for object recognition using the proposed descriptors and different quaternion moment invariants: (a) and (b) correspond to Gaussian noise, (c) and (d) to smooth distortion, (e) and (f) to salt-and-pepper noise 
Table 1

The average RMS error and maximum error of estimated angles

\begin{tabular}{|c|c|c|c|c|c|c|c|}
\hline \multirow{2}{*}{ Moments } & \multirow{2}{*}{ No. } & \multicolumn{2}{|c|}{ error } & \multirow{2}{*}{ Moments } & \multirow{2}{*}{ No. } & \multicolumn{2}{|c|}{ error } \\
\hline & & $e_{1}$ & $e_{2}$ & & & $e_{1}$ & $e_{2}$ \\
\hline \multirow{3}{*}{ QBFMs } & 21 & $0.0612^{\circ}$ & $0.1277^{\circ}$ & \multirow{3}{*}{ QZMs } & 20 & $0.0744^{\circ}$ & $0.1508^{\circ}$ \\
\hline & 28 & $0.0543^{\circ}$ & $0.1181^{\circ}$ & & 30 & $0.0639^{\circ}$ & $0.1348^{\circ}$ \\
\hline & 36 & $0.0515^{\circ}$ & $0.1097^{\circ}$ & & 36 & $0.0576^{\circ}$ & $0.1199^{\circ}$ \\
\hline \multirow{3}{*}{ QPZMs } & 21 & $0.0660^{\circ}$ & $0.1302^{\circ}$ & \multirow{3}{*}{ QOFMMs } & 21 & $0.0878^{\circ}$ & $0.1810^{\circ}$ \\
\hline & 28 & $0.0589^{\circ}$ & $0.1220^{\circ}$ & & 28 & $0.0859^{\circ}$ & $0.1747^{\circ}$ \\
\hline & 36 & $0.0562^{\circ}$ & $0.1155^{\circ}$ & & 36 & $0.0830^{\circ}$ & $0.1707^{\circ}$ \\
\hline
\end{tabular}

